\title{
Reconnection and Waves: A Review with a Perspective
}

\author{
M. Fujimoto $\cdot$ I. Shinohara $\cdot$ H. Kojima
}

Received: 17 September 2010 / Accepted: 22 June 2011 / Published online: 28 July 2011

(C) The Author(s) 2011. This article is published with open access at Springerlink.com

\begin{abstract}
This review is intended to help prepare a new stage of wave studies in the context of magnetic reconnection. Various results that have accumulated would not let the twodimensional, steady and laminar magnetic reconnection to remain as the standard model. Emphasis on three-dimensional, temporally varying, and turbulent effects is growing and this fact tells that the effects of waves in various frequency ranges deserve further attention in the context of magnetic reconnection. In this review, by setting a perspective, selected recent topics are reviewed and the ways in which these can be viewed as the stepping stones towards a new research horizon of magnetic reconnection are discussed.
\end{abstract}

Keywords Electron dynamics in reconnection - Electron-ion coupling in reconnection · $\mathrm{X}$-line interaction with the surrounding environment

\section{Introduction}

Magnetic reconnection is certainly one of the most studied processes in space plasma physics. Magnetic reconnection is capable of explosive conversion of magnetic energy that has accumulated quietly prior to the onset of the process. In a fluid-type description, the magnetic energy is converted into thermal as well as bulk flow energies of the plasma gas. In the collisionless environment that prevails in space plasmas, a part of the released energy is utilized for producing very energetic (non-thermal) particles. While magnetic reconnection is a large-scale (MHD-scale) phenomenon as a whole, the process requires non-idealMHD effects to be operative in its engine part and accompanies sub-MHD-scale dynamics in various other parts.

Highly dynamic and multi-scale aspects of magnetic reconnection naturally let studies on associated waves to emerge with the following perspectives: (1) The highly dynamic

M. Fujimoto (凶) - I. Shinohara

ISAS, JAXA, Sagamihara, Japan

e-mail: fujimoto@stp.isas.jaxa.jp

H. Kojima

RISH, Kyoto University, Kyoto, Japan 
phenomenon in a collisionless plasma is expected to produce unstable particle distribution functions, such as a beam superposed on a stable component. Plasma wave data are useful tools to sense (sometimes remotely) the generation of such an unstable distribution function in the course of a reconnection event. (2) Waves confined to a small region are often activated as the local plasma responds to larger-scale dynamics that surrounds the region. Some of the waves are excited not just in a parasitic way but would have a non-linear feed-back effect on the larger-scale parent process. A typical argument in this line is that wave-induced dissipation is responsible for "anomalous resistivity" needed to initiate and maintain magnetic reconnection running.

This review is motivated by the idea that studies on waves associated with magnetic reconnection deserve further attention. Various studies have shown that reconnection in nature does not proceed in a steady and laminar manner. This growing recognition also enhances the interest in wave phenomena associated with reconnection because wave data enable us to prove temporal variations at multiple scales. This review is not at all intended to achieve the impossible task of fully covering the literature related to the topic. Instead, we have tried to select the items that would be regarded as the stepping stones towards a new stage of magnetic reconnection research. We have categorized the topics according to their temporal/spatial scales. The three sections that follow this Introduction discuss waves at the electron scale, waves at the electron-ion hybrid scales, and waves at ion and MHD scales, respectively. In a traditional framework, low frequency waves at ion and MHD scales have not necessarily been discussed in the context of reconnection. This is presumably because the dissipation mechanism in a diffusion region has been the focus in the previous discussion. One of the arguments to be put forward in this review is that understanding the physics of diffusion region is not all that matters. Various multi-scale phenomena are induced in the surrounding region as a diffusion region ejects a pair of plasma jets outward, which certainly play various roles in producing the consequences that entitle magnetic reconnection to be one of the most attractive processes in space plasma physics.

To motivate the readers who have not necessarily been familiar with wave data, let us show the data from the Geotail's best reconnection region crossing event. This event took place in the near Earth plasma sheet at $X \sim-27 \mathrm{R}_{\mathrm{e}}$ (GSM coordinates) when a moderate substorm was recorded on the ground. In Fig. 1, (a) the magnetic field, (b) the omnidirectional energy-time spectrogram of ions and electrons, (c) the ion moment, and the frequency-time spectrogram of (d) magnetic and (e) electric field waves for 1050-1100 UT are shown. Geotail approached the neutral sheet and observed an onset of tailward plasma flow with southward $B_{z}$ at 1051 UT. A flow reversal earthward was recorded at around 1055:40 UT, and $\mathrm{B}_{\mathrm{z}}$ turned northward simultaneously. A pair of ion distribution functions, one sampled just prior and the other just after the flow reversal, are composed of the lobe component that is being entrained into the current sheet and the beam component that is being ejected out from the X-line. The flow reversal is realized as the direction of the beam component changes: The lobe component is seen to remain mostly the same. In addition to these ion kinetic feature, Fig. 1b shows that electrons are also energized $(>1 \mathrm{keV})$ in the flow reversal region. These are the characteristic signatures seen in data obtained by a spacecraft making a close encounter with a diffusion region (Nagai et al. 1998, 2001; Shinohara et al. 1998). Nagai et al. (2011) also shows that, associated with energetic electrons, a super Alfvénic cross-tail electron bulk flow $\left(V_{e y}\right)$ that would sustain the intense current density is present.

Now the question is if there are active waves in the near-diffusion region. Figure 1c shows the frequency-time spectrograms of the magnetic field in the frequency range of 1$32 \mathrm{~Hz}$. Three panels indicate the Fourier spectra for polarized components of BR (right-hand 
(a)

May 15, 2003 GEOTAIL MGF LEP PWI

(b)
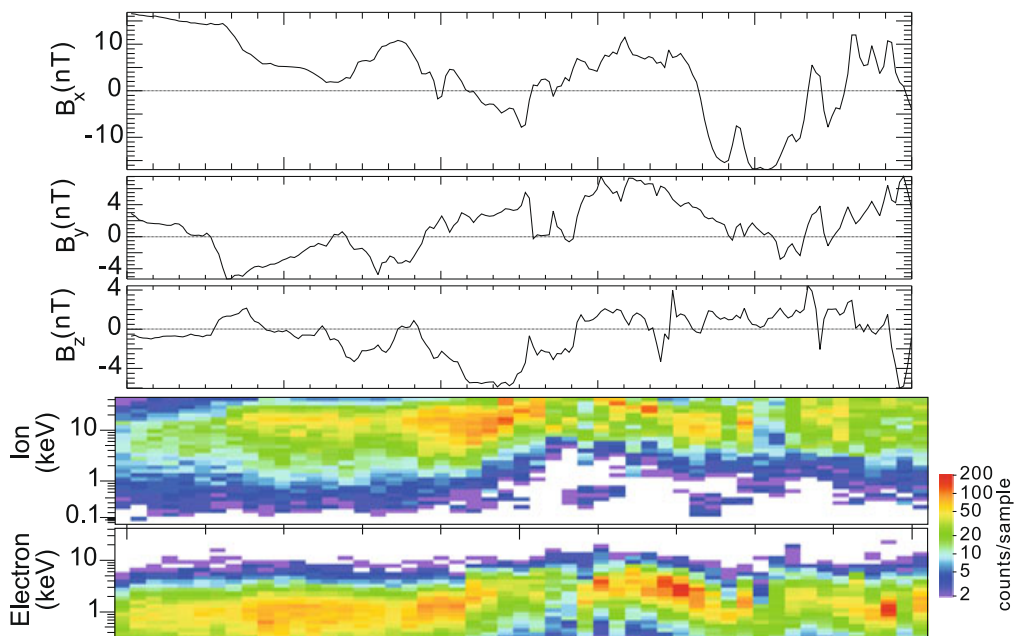

(c)
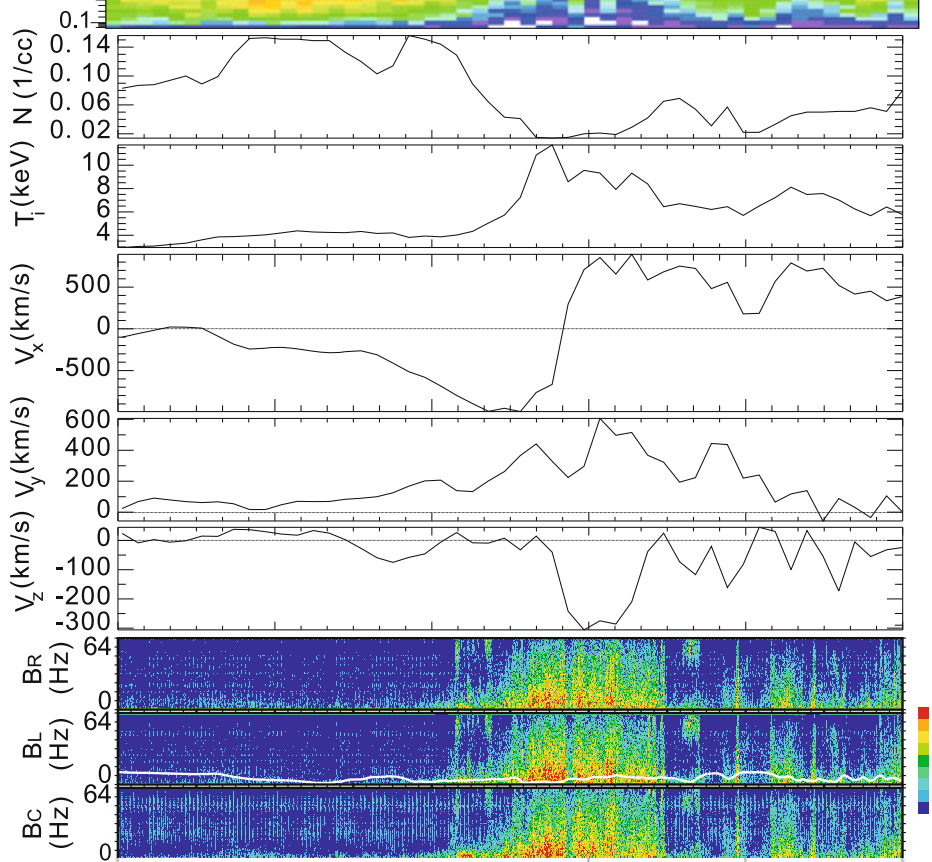

(d)

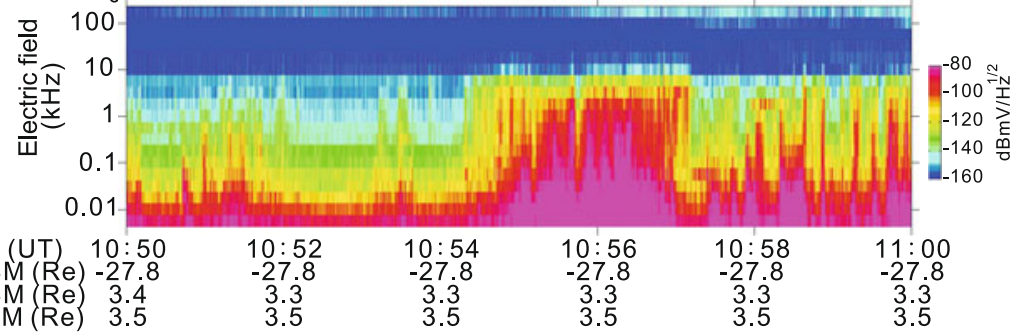

Fig. 1 The data from the 10 min interval in the Geotail's best reconnection region crossing event are shown. Intense wave activities shown in panels (c) (ion-electron hybrid frequency range) and (d) (electron frequency range) accompany the flow reversal (an X-line crossing) at 1055:40 UT 
polarized), BL (left-hand polarized), and BC (compressional), respectively. Intense wave activities in the lower-hybrid frequency range (shown by the white line) are observed around crossings of the neutral sheet. These signatures of the magnetic field fluctuation are quite similar to those reported in Shinohara et al. (1998), which are reviewed in a later section. Figure $1 \mathrm{~d}$ is the frequency-time spectrogram of the electric field component obtained from the Multi-Channel Analyzer (MCA) of the Plasma Wave Instrument (PWI). The intense emission up to a few $\mathrm{kHz}$ can be seen around the flow reversal at 10:55:40. Unfortunately, no waveform capture data are available during this interval. According to a series of snapshots of frequency-intensity spectrum (not shown), however, we can reasonably infer that solitary waves must have appeared intermittently during this period.

This event clearly shows that electron- and electron-ion hybrid scale waves are excited in the reconnection region that drives large-scale dynamics as a whole. At the same time, it should be worth pointing out that the wave activities are more or less absent right at the flow reversal, namely, the X-line. That is, the data do not necessarily fit with the conventional idea in which plasma waves are the agent to generate "anomalous resistivity" at the X-line.

As mentioned in a previous paragraph, wave-induced dissipation at an X-line that is responsible for "anomalous resistivity" needed to initiate and maintain magnetic reconnection has been one of the main issues in the "reconnection and wave" theme. Here let us discuss briefly if this concept is really needed. In a two-dimensional geometry where the reconnecting magnetic field is in the $x$-direction and the current sheet normal is in the $z$-direction (as in the magnetotail), what is needed is the $y$-directed electric field $E_{y}$. Since there is intense $y$-directed current density $j_{y}$, one can very easily see that anomalous resistivity would make $E_{y}$ to be present. Furthermore, one would be naively inclined to think that the intense current density would give rise to a current driven instability having a wave vector in the $y$ direction which facilitates ion-electron momentum exchange ("anomalous resistivity"). In two-dimensional particle simulations, however, where the instability mode is not available, the electric field $E_{y}$ is present and is maintained by off-diagonal electron pressure terms (e.g., Hesse et al. 2002). Viewed from a different perspective, $E_{y}$ is needed to deflect and accelerate the electrons which are in-flowing along the $z$-axis towards the $y$-direction such that the intense current density at the X-line $j_{y}$ is secured. Here one can clearly see that "resistivity" (ion-electron momentum exchange) is not necessary. Furthermore, it has been shown that the off-diagonal pressure terms would be formulated in terms of the electron flow pattern characteristics around an X-line (Kuznetsova et al. 2007). The off-diagonal electron pressure terms become effective only when the characteristic scale length is reduced down to an electron scale. While one argue that, in a fully three-dimensional situation, a currentdriven instability would set-in to trigger reconnection before such a minuscule structure is formed, a promising candidate of this nature has not been identified to date. On the other hand, as will be shown later, there seems to be a good chance that turbulence-like behavior will emerge once an electron-scale current layer is formed in a fully three-dimensional situation. It is still a matter of question if the bulk effects of the turbulent reconnection region should be characterized by the term "resistivity".

\section{Waves at Electron Scales}

Recent observations in the Earth's magnetosphere have shown the emergence of thin electron-dominated current sheets in association with magnetic reconnection (e.g., Mozer et al. 2002; Asano et al. 2004; André et al. 2004; Wygant et al. 2005; Nakamura et al. 2006; Chen et al. 2008a, 2008b). Electron dynamics therein, however, is not resolved well 
enough by particle data of today. High-frequency waves at an electron scale are useful in that they enable us to probe the electron dynamics. Recent plasma wave observations have revealed the electron features seen in the crucial regions of reconnection. The examples of plasma waves driven by electron scale dynamics coupled with large-scale magnetic reconnection are electrostatic solitary waves (ESW) in the dayside magnetopause (Matsumoto et al. 2003) and in the magnetotail (Farrell et al. 2002; Deng et al. 2004; Cattell et al. 2005; Khotyaintsev et al. 2010), upper hybrid waves in the diffusion region (Farrell et al. 2002), and whistler-mode waves in the dayside magnetopause (Deng and Matsumoto 2001) and in the magnetotail (Petkaki et al. 2006; Wei et al. 2007). The contributions of these plasma waves to the whole reconnection picture remain unclear. However, possible roles of plasma waves showing the electric field amplitudes of more than a few $\mathrm{mV} / \mathrm{m}$ (Matsumoto et al. 2003; Farrell et al. 2002; Cattell et al. 2005; Khotyaintsev et al. 2010) should not be ignored before a detailed inspection is made.

Farrell et al. (2002) reported observations of ESWs and upper hybrid waves near an electron diffusion region. They showed amplitude modulation such that they were observed in an alternating manner. These waves have also been observed around the plasma sheet boundary, which is far from an X-line. ESW will be discussed in more detail later. Regarding the upper hybrid waves at the plasma sheet boundary layer, Kojima et al. (1997b) observed modulated waveforms that contained a mixture of parallel and perpendicular polarization relative to the ambient magnetic field. In the magnetotail, the upper hybrid frequency is almost equal to the electron plasma frequency because the electron cyclotron frequency is much lower than the electron plasma frequency. Therefore polarization is useful information to categorize the wave mode. The parallel-polarization waves reported by Kojima et al. (1997b) would indicate the presence of Langmuir waves rather than the upper hybrid waves. The observations by Kojima et al. (1997b) and Farrell et al. (2002) showed significant difference in the amplitude of the upper hybrid waves. While the processes for generating the above upper hybrid waves of two different amplitudes may not necessarily be the same, these upper hybrid waves are observed in association with electron heat flux/beam features. The amplitude of the order of a few tens of $\mathrm{mV} / \mathrm{m}$ reported by Farrell et al. (2002) is unexpectedly large. If the upper hybrid waves of such large amplitudes are common near the X-line, their effects, which are unidentified as of now, deserve to be studied.

Recent spacecraft observations and computer simulations have revealed that reconnection is accompanied by another type of large amplitude electrostatic wave. This is ESW, which was initially observed by Geotail in the plasma sheet boundary layer (Matsumoto et al. 1994b). ESW clearly indicates that the electron-wave interaction proceeds well into the non-linear stage. The robust identification of the ESW requires wave-form observations that were made for the first time by the "wave-form capture receiver" (WFC) of the PWI onboard Geotail (Matsumoto et al. 1994a).

Before the waveform information was available, the wave mode now known as ESWs had been recognized by its broadband electrostatic noise (BEN) feature in a frequency-time diagram. Figures $2 \mathrm{a}$ and $2 \mathrm{~b}$ show the frequency-time spectrogram of BEN observed in the PSBL at $X \sim-95 \mathrm{R}_{\mathrm{e}}$ (Kojima et al. 1997a). The spectra shown in Figs. 2a and 2b were generated from the electric field data of the sweep frequency analyzer (SFA) and multichannel analyzer (MCA) onboard Geotail, respectively. Intense power spectra extending all the way up to the electron plasma frequency $(\sim 2 \mathrm{kHz})$ appeared in the interval 13:11-13:15 (UT). Since BEN in the PSBL had been observed in good correlation with high-energy ion beams, many theoretical studies had attempted to relate BEN to ion beam instabilities. These attempts had a difficulty in explaining the broadness of BEN spectra, which extend over the frequency range from lower hybrid frequencies up to electron plasma frequencies. On the 
Fig. 2 Frequency-time spectrograms of (a) SFA and (b) MCA in the different time scales. (c) Snapshot of the waveforms for the broadband electrostatic noise at 1310:35.166 (UT): From Kojima et al. (1997a)

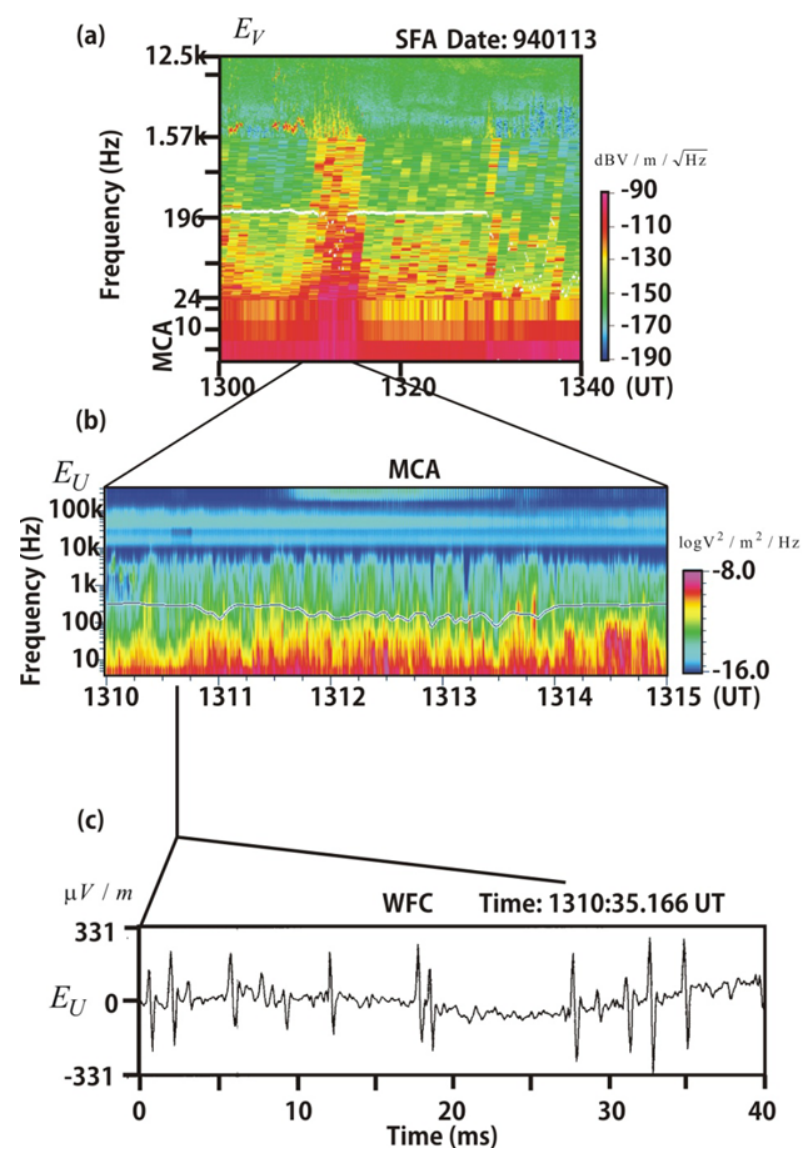

other hand, Parks et al. (1984) demonstrated the relationship between BEN and electron beams based on observations by the ISEE-1 spacecraft. Further detailed analyses of the relationship between BEN and electron velocity distributions were conducted by Onsager et al. (1993), who showed that BEN can be observed in the outer PSBL where electron beams were present but energetic ions were absent.

Figure 2c shows the waveform observed by WFC at 13:10:35.166, when BEN was present in the frequency-time diagram. The waveforms turned out to be completely different from those that had been expected by many scientists who had studied BEN. The waveform is made up of a series of bipolar signatures, with each of them characterized by solitary spikes composed of a half-sinusoid-like cycle followed by a similar half-cycle of the opposite sign. Matsumoto et al. (1994b) concluded that each bipolar pulse of an ESW is an isolated potential structure flowing along the ambient magnetic field. PWI onboard Geotail cannot identify the polarities or flow speeds of electrostatic potentials. However, the features of the ESW in the PSBL indicate that the isolated potential is an electron hole that is charged positively. The timescale, such as the pulse widths, is on the order of ms, and the amplitude is relatively small, on the order of a few hundred $\mu \mathrm{V} / \mathrm{m}$. This time scale is of the order of the electron plasma frequency ( $\sim \mathrm{a}$ few $\mathrm{kHz})$, and the small amplitude does not enable ions to interact with the pulses. 
Fig. 3 The scatter plot showing potential energies versus spatial scales of ESWs observed by Geotail. The symbols of diamonds and crosses mean earthward and tailward travelling ESW, respectively. The statistical property supports the bump-in-tail instability origin of the ESWs. From Kojima et al. (1999)

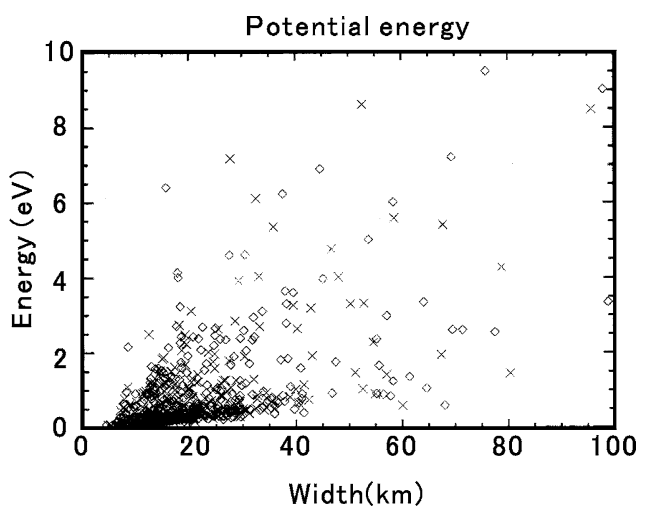

Matsumoto et al. (1994b) and Omura et al. (1994) demonstrated that the nonlinear evolution of electron beams led to the formation of isolated potential structures. Furthermore, Omura et al. (1996) concluded that the bump-on-tail instability was the most plausible mechanism for the ESWs in the PSBL. The beam velocity should be higher than that of the background electron thermal velocity. Such a situation can be realized commonly in the PSBL, which is a corridor for energetic electrons accelerated at a reconnection region. Figure 3 shows a scatter plot showing potential energy versus spatial scale of ESWs observed by Geotail in the geomagnetic tail (Kojima et al. 1999). As shown, the ESW potential energies were mostly below $4 \mathrm{eV}$. The ESW potential was very weak relative to the background thermal energy. On the other hand, the potential flow velocity was comparable to that of an electron at a few hundred $\mathrm{eV}$. The ESW potential traps beam electrons as well as background electrons that are distributed around the beam velocity. The trapping effect results in the diffusion and thermalization of the beam and the background electrons. ESWs discovered by Geotail in the magnetotail region are so-called fast ESWs (fast electron holes).

Representative studies that followed these pioneering works on fast ESWs are as follows: Umeda et al. (2002) performed a two-dimensional electrostatic simulation of the bump-ontail instability. By adding the dimension perpendicular to the magnetic field, they showed that coupling to the lower-hybrid mode appears in the non-linear stage of the instability. Fujimoto and Machida (2006) showed that ESWs via the bump-on-tail instability are indeed excited along the separatrices in a large-scale two-dimensional full-particle simulation of magnetic reconnection.

Buneman instability has also been known to produce solitary waves. A simulation study by Drake et al. (2003) revived significant interest in this direction. In a three-dimensional full-particle simulation of reconnection with a strong guide field, it was shown that ESWs are generated at the X-line via Buneman instability, which is made unstable by the strong current density (large bulk flow speed difference between ions and electrons) at the site. Unlike the fast ESW generated by the bump-on-tail instability, ESW via Buneman instability is slower (slow ESW) and can couple ions and electrons to reduce the difference in the bulk flow speed and to produce effective resistivity. While it is unlikely that Buneman ESW is necessary to maintain reconnection in the simulation, this "anomalous resistivity" aspect has certainly helped to broaden the interest in the topic. Furthermore, the instability condition requires a very large velocity difference between ions and electrons, and is far tougher to achieve than that required for the bump-on-tail instability. While such a large difference in the bulk flow speed was achieved in the course of the simulation, whether reconnection in nature indeed involves such a thin current sheet is a very interesting question. 
At this stage, our concern is whether or not the generation mechanism of the ESW observed by Geotail is the same as that of the ESW discussed by Cattell et al. (2005). Cattell et al. (2005) claimed that the ESWs they detected in Cluster-II data were produced by Buneman instability, because the traveling velocity of the ESW was much lower than the electron beam velocity. Indeed, fast ESWs could not have been detected by the instrument onboard Cluster-II due to instrumental limitations. On the other hand, as stated above, the ESW observed by Geotail can be well understood in terms of the bump-on-tail instability. The amplitudes of the ESWs detected by Cluster-II were several orders of magnitude larger than those discovered by Geotail. The spatial scale was about an order of magnitude smaller, resulting in an order of magnitude difference in the associated electrostatic potential.

A recent simulation study by Che et al. (2010) demonstrated that two kinds of ESWs can be generated in the dissipation region during $3 \mathrm{D}$ magnetic reconnection with a guide field. The simulation study used an initial current density that was so strong as to be unstable to Buneman instability. From this initial condition, ESWs emerged in a transient manner. Che et al. (2010) concluded that the simultaneous existence of two kinds of ESWs having two different phase speeds enables electron scattering over a very large range of velocity space. This would not have been possible by either one of them alone. In connecting this kind of simulation studies to observations, the most significant problem would be to think of the mechanism that produces and maintains such a strong current density which is unstable to Buneman instability. Larger-scale dynamics surrounding the region of interest may continuously pinch a current sheet significantly, and then may maintain the unstable conditions for a long enough time interval so that a spacecraft would have a good chance of encountering the product of the instability. Simultaneous multi-scale observations by a well-coordinated spacecraft fleet would be required to address this issue.

Ergun et al. (2009) reported the first direct observations of parallel electric fields carried by double layers (DLs) in the plasma sheet of the Earth's magnetosphere. DLs are observed during bursty bulk flow events and in the plasma sheet boundary layer, and are very likely related to magnetic reconnection. These DLs are analogous to those reported in the auroral region, and have been detected in association with large amplitude ESWs. DLs are another manifestation of non-linear electron dynamics that is operative in association with magnetic reconnection.

\section{Waves at Ion-Electron Hybrid Scales}

The wave modes in this frequency/wavelength range enable ion-electron coupling and thus can be the agent for dissipation. This is the reason that this frequency range has been one of the focal points of the previous studies on waves associated with magnetic reconnection.

The explosive nature of reconnection onset implies that there should be some thresholds above which non-ideal effects set in to trigger the process. A number of microscopic turbulent processes resulting from current-driven instabilities have been inspected from this point of view. Here we list the instabilities that have been proposed to explain the "anomalous resistivity" at the neutral sheet: the ion acoustic instability (Coroniti and Eviatar 1977), the electron cyclotron drift instability (Haerendel 1978), the lower hybrid drift instability (Huba et al. 1977), and the cross-field streaming instability (Lui et al. 1990).

Since the ion acoustic wave is a normal mode in an unmagnetized plasma, the ion acoustic mode can be excited even in the field free magnetic null point. However, in a usual magnetotail condition where the ion temperature is higher than that of electron, the ion acoustic instability can be excited only in an extremely thin current sheet. Such a thin current sheet has never been observed. The electron cyclotron drift instability also requires an 
extremely small current sheet thickness. The most plausible instability which can produce the anomalous resistivity is the lower hybrid drift instability (LHDI). LHDI was first invoked by Krall and Liewer (1971) for pinch devices. LHDI is driven by a diamagnetic drift and can result in substantial resistivity even in a low drift regime (Davidson et al. 1977). However, Huba et al. (1980) showed that LHDI was strongly stabilized in a high beta region of a neutral sheet and thus was localized at the edges remote from the center of a current sheet. Computer simulations of LHDI confirmed this difficulty for the lower hybrid turbulence to permeate into a neutral sheet (Tanaka and Sato 1981; Winske and Liewer 1978; Brackbill et al. 1984). The conclusion from these theoretical considerations was that there seemed to be no suitable instability to supply the dissipation at the center of a current sheet.

Spacecraft observations show evidence of LHDI in the magnetotail (Gurnett et al. 1976; Huba et al. 1978; Cattell et al. 1982; Okada et al. 1994; Shinohara et al. 1998). Most observations reveal that during geomagnetically quiet times BEN and magnetic noise bursts (MNB) are suppressed near a neutral sheet (Scarf et al. 1974; Gurnett et al. 1976; Cattell et al. 1982). During geomagnetically active times, MNBs are frequently observed at a neutral sheet (Scarf et al. 1974; Gurnett et al. 1976; Coroniti and Eviatar 1977). Cattell and Mozer $(1986,1987)$ found in the data from ISEE-1 that large-amplitude electric field waves existed right at a neutral sheet and that these waves would be relevant to the formation of the near-Earth neutral line. It was also shown that the frequencies of electric field waves observed around a neutral sheet were well correlated with the lower hybrid frequency. Thus, these observations are consistent with a scenario that LHDI is destabilized when the plasma sheet thins prior to substorm onsets and that LHDI supplies the anomalous resistivity around the neutral sheet necessary to trigger reconnection. However, since the lowest frequency range of these early wave observations was higher than tens of $\mathrm{Hz}$, which is above the lower hybrid frequency around a neutral sheet, previous observations could not provide sufficient information about LHDI in the high beta region.

Geotail is the first spacecraft that successfully made comprehensive observations of the electromagnetic field in the lower hybrid frequency range. The search-coil magnetometer and the double probe experiment onboard Geotail provided us with the ELF range (1-32 Hz) magnetic and electric waveform data. Okada et al. (1994) found quasi-monochromatic waves in the lower hybrid frequency range in the PSBL associated with fast plasma flows. The wave vectors were directed perpendicular to the local magnetic field. Shinohara et al. (1998) examined the instability condition of the low-frequency $(1-10 \mathrm{~Hz})$ electromagnetic turbulence observed in the close proximity of reconnection onset sites. They showed that the observed characteristics of the electromagnetic turbulence were in good agreement with the linear properties of LHDI. Shinohara et al. (1998) also evaluated the anomalous transport by the electromagnetic turbulence. Using the quasi-linear theory it was shown that the observed wave energy density agreed well with the expected saturation level. It was also shown that the estimated anomalous resistivity is $\sim 10$ orders of magnitude greater than the classical resistivity. The number, however, was smaller than the one that drives a quickly growing resistive tearing mode. Cluster-II observations also confirmed that lower hybrid wave turbulence is excited at the high-latitude edges of the current sheet where a density gradient is present (Zhou et al. 2009a, 2009b). A similar feature was reported from a magnetopause case (Bale et al. 2002). A localized lower hybrid wave activity at the edges of a current sheet was seen in a laboratory experiment as well (Carter et al. 2002).

Is LHDI that can be effective only at the edges of a current sheet totally useless in reconnection triggering? Recent numerical simulation studies revealed a possible role that the edge-LHDI might play in triggering magnetic reconnection. Several authors (Scholer et al. 2003; Ricci et al. 2004; Shinohara and Fujimoto 2005; Tanaka et al. 2005) have reported that 
Fig. 4 Three-dimensional full particle simulation results showing the effects of the edge-LHDI. $E_{y}$ on the $y-z$ plane shows that the LHDI activity is confined the edges. The perp/para electron temperature ratio on the $x-z$ plane indicate that the non-local impact of the LHDI activity is to create an electron temperature anisotropy (with the perpendicular temperature being $\sim 1.2$ times that of the parallel) at the center of the current sheet

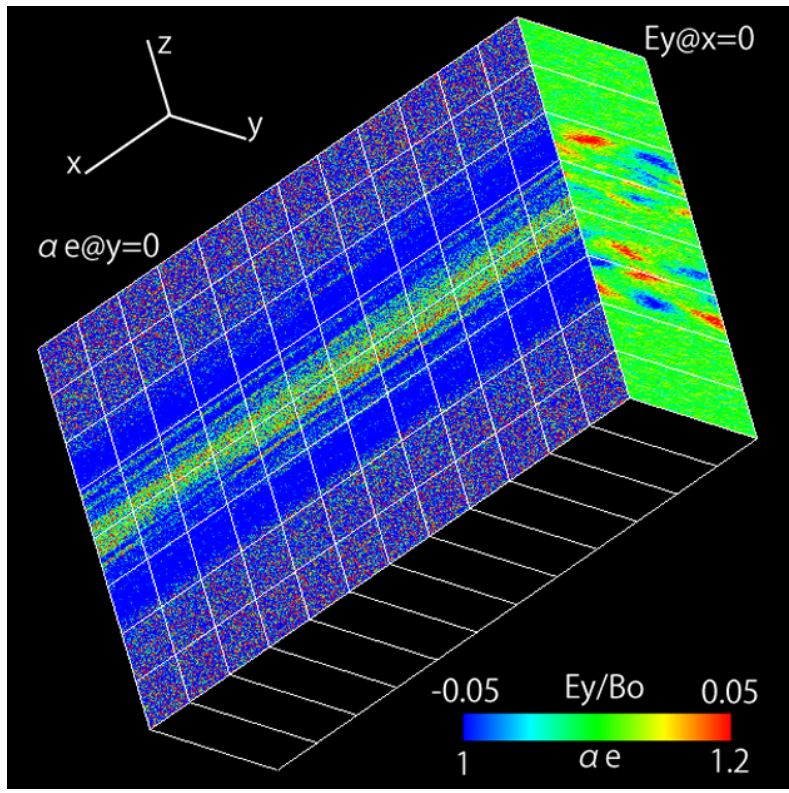

quick triggering took place in an ion-scale current sheet with a help from the nonlinear evolution of LHDI. The new scenario for Quick Magnetic Reconnection Triggering (QMRT) is as follows (Shinohara and Fujimoto 2005): (1) The cross-field current at the edges of a current sheet is strongly reduced during the nonlinear phase of LHDI. (2) The current reduction at the edges makes the spatial gradient of the magnetic field become less steep, which in turn makes a part of the magnetic field at the edges penetrate toward the neutral sheet. (3) The inductive electric field associated with the change in the magnetic field profile accelerates meandering electrons around the neutral sheet. (4) The electron current density around the neutral sheet is enhanced and a thin electron current layer is formed. (5) The increased number of meandering electrons in a thin current sheet strongly enhances the tearing growth rate. The steps (4) and (5) above can be modified by noting that electron temperature anisotropy enhances the growth rate of the tearing mode substantially (Ricci et al. 2004; Daughton et al. 2004). (4') The electron temperature anisotropy (with the perpendicular temperature being larger than the parallel) develops around the neutral sheet. (5') The growth rate of the tearing mode is elevated substantially. It was shown that the initial current sheet thickness determined which scenario will be followed (Tanaka et al. 2005). For a thicker current sheet, the electron temperature anisotropy develops near the neutral sheet (Fig. 4). Then the anisotropy-boosted tearing mode is driven unstable (Type-II QMRT).

In parallel to these numerical simulation efforts, quest for electromagnetic fluctuation in the neutral sheet continued. The electromagnetic lower hybrid waves as well as the electrostatic lower hybrid waves have been detected in the MRX laboratory experiment (Carter et al. 2002; Ji et al. 2004). Ji et al. (2004) showed that the magnitude of the electromagnetic lower hybrid fluctuation at the center of a reconnecting current sheet was positively correlated with an enhancement in reconnection rate, while the electrostatic fluctuations at the edges did not play an essential role. The electromagnetic waves were identified as right-hand polarized whistler waves, propagating obliquely to the reconnecting field, originating in the modified two-stream instability. However, how to excite the modified two-stream instabil- 
ity has not been necessarily clear. The excitation of the instability may well happen after reconnection has matured but not in the triggering phase.

In contrast to the previous linear analyses that were based on the local approximation, global eigen-mode analyses of thin Harris current sheets have been carried out by Daughton (1999, 2003) and Yoon et al. (2002). Daughton (2003) found that unstable modes with a significant electromagnetic component would provide a fluctuating component in the center of a current sheet. The mode has a longer wavelength than the electrostatic mode confined to the edges. Daughton (2003) also demonstrated in a full particle simulation that the electromagnetic LHDI showed non-negligible amplitude in the central current sheet. Electromagnetic fluctuations in the lower-hybrid frequency range have been detected at the center of a thin current sheet in a laboratory experiment (Carter et al. 2002; Ji et al. 2004) and in space as well (Shinohara et al. 1998; Bale et al. 2002).

The topic now touches on the issue of the stability property of a non-Harris current sheet. It goes without saying that the Harris current sheet has been the "standard" model for theorists to inspect kinetic aspects current sheet dynamics. Meamwhile, multi-spacecraft observations have shown that this is not necessarily the choice of the nature. Using multi-satellite Cluster magnetic field data, Asano et al. (2005) statistically examined how often a nonHarris type current sheet appeared in the magnetotail. Current density profiles inside thin current sheets having half-thickness of $\sim 1500 \mathrm{~km}$ were examined. It was found that a current density profile having an off-equatorial maximum (that is, a bifurcation current sheet) was frequently detected. It was also found that many of the center-peaked current sheets exhibit an intense current filament embedded inside a broader layer, which was another non-Harris feature seen in the real situation.

These observations stimulated theoretical studies that constructed a Vlasov equilibrium of a thin current sheet having a bifurcated structure. Here we introduce an example by Sitnov et al. (2003). Presented in the paper was a generalization of the Harris current sheet equilibrium that reproduced a bifurcated current sheet. While the Harris model was constructed by the Hamiltonian and a component of the canonical momentum of particles, both of which are the constants of motion, the ion distribution function in the new model had dependence on the quasi-adiabatic invariant of the ion motion across the current sheet (hereafter denoted by $I_{z}$ ) and assumed ion temperature anisotropy outside the current sheet. Bifurcated current sheets appeared in the case of small ion anisotropy with the perpendicular temperature being larger than the parallel. One of the key features of the Sitnov-Guzdar-Swisdak model (SGS) was the presence of the non-Maxwellian ion distributions with some degree of nongyrotropy, which was sustained by meandering ions. The non-Maxwellian part arose directly from the dependence of the distribution function on $I_{z}$ and became significant when the current sheet thickness becomes comparable to the thermal ion gyroradius.

This SGS model was used in a data analysis study by Zhou et al. (2009a, 2009b). In their paper the ion distribution function data obtained in a thin current sheet prior to an onset of a substorm were studied. Two THEMIS spacecraft were located in the close proximity to what would turn out to be the site of an $X$-line $\left(X \sim-20 \mathrm{R}_{\mathrm{e}}\right)$. Both spacecraft detected ion distribution functions showing mushroom-shaped structures with clear nongyrotropic features. The nongyrotropic feature possibly indicated that the mushroom-shaped component was unmagnetized in the current sheet, which was possible only if the gyroradii of these ions were comparable with the current sheet thickness. Since the mushroom-shaped ions were seen to be superposed on a cooler Maxwellian component, reproduction of the observed distribution function was tried with a distribution function composed of the SGS model and the Harris-type model. After a successful reproduction of the observed ion distribution function, reconstruction of the current sheet structure was performed. The mushroom-shaped ions that 
had meandering orbits across the neutral sheet were found to remarkably alter the current sheet profiles and therefore would have played an important role in the formation of the thin current sheet. The reconstruction also indicated that the spacecraft location where the ion distribution was sampled was at the edge of the current sheet located $\sim 1000 \mathrm{~km}$ away from the neutral sheet. While this is certainly a nice piece of work, one issue which cannot necessarily be taken as proposed is the assumption that the cooler Maxwellian component continues to be present all the way down to the neutral sheet. A different story that would emerge if one has the temporal sequence of current sheet thinning in mind would involve ion heating near the neutral sheet and an embedded current filament sustained by electrons that drift under a pair of vertical electric field directed towards the neutral sheet. Synergy between multi-spacecraft observations and a particle simulation would enable a researcher to pursue this kind of study with a temporal sequence in mind.

\section{Waves at Ion-Scales and at Lower Frequencies}

The standard magnetic reconnection picture has been a two-dimensional one in which a single steady X-line drives the process in a laminar manner. One can easily see that there is a wide open area beyond this standard picture. Here we would like to pick up the topics that would promote the expansion of the research horizon. The keywords are: threedimensional effects, turbulence, and interaction with the surrounding media. Low frequency waves, which would not have been included in the "reconnection and waves" theme in a traditional framework, do play roles in the situations highlighted by these keywords.

Because of the huge computational resources required, most of the full particle simulations of magnetic reconnection have been two-dimensional. An interesting effect of QMRT was discovered when the minimum freedom in the third dimension to allow LHDI was included. Much larger three-dimensional simulation results by Daughton et al. (2011) showed that an even more drastic change in the way we understand the process might be needed. The system size in the third dimension was as large as 70 ion inertial length. The simulated case had a guide field, and, as is well known in two-dimensional cases, extended electron-scale current sheets formed nonlinearly during the onset of reconnection along the separatrices. Whereas the current layers were stable in two-dimension cases, the three-dimensional case showed a new scenario in which flux ropes were generated via the tearing instability in the highly extended thin current layer. The electron-scale current sheets were seen to continually reform and break up into filaments. The reconnection region was filled with turbulence that was continually self-generated. These results have immediate implications for observation studies of magnetic reconnection. The event search to date, which has been based on an idealized two-dimensional geometry, must have missed many events showing more complex behavior.

Chaston et al. (2009) has demonstrated via multipoint observations from the Cluster spacecraft of a guide field reconnection case that kinetic Alfvén waves (KAWs) may play an important role in facilitating magnetic reconnection. The waves were identified in the close proximity of an X-line and were found to radiate outwards from the diffusion region oblique to the magnetic field. It was argued that the ions in the proximity of the X-line would be subject to spatial diffusion via a coupling between the gradient drift of the ions and the ion motion in the wave field (Johnson and Cheng 1997). The estimated transport efficiency was comparable to the transport by the observed inflow towards the X-line. KAWs are inherently of a three-dimensional nature. The argument presented here suggests the possibility that non-ideal effects in the diffusion region may be sustained by the low-frequency waves that cannot be included in the conventional two-dimensional picture. 
Low frequency waves are radiated out of the reconnection region. Eastwood et al. (2009) presented observations of electromagnetic fluctuations within a magnetic reconnection region (ion diffusion region) under zero guide field. The localization of the spacecraft within the ion diffusion region was done by inspecting the magnetic and electric field components, which provided evidence that Hall-MHD physics was operative. Turbulent cascades in both the electric and magnetic field fluctuations were inferred from the power law scaling, and comparison with the dispersion relation indicated that the energy injected by the reconnection exhaust propagates along the whistler wave branch. It was proposed that the dispersive waves might be important in particle acceleration processes. Chaston et al. (2005) reported that a reconnection region on the dayside magnetopause (with guide field) was the source of kinetic Alfvén waves that carried substantial amount of Poynting flux into the ionosphere leading to the up-welling of oxygen ions. These two studies suggest that the energy carried by low frequency waves outward from a reconnection region can have significant remote effects.

In a transient reconnection picture, an X-line starts to emanate a jet and the jet-front propagates into the plasma at rest surrounding the X-line. Compressional interaction between the jet front and the rest-plasma ahead is naturally expected when one bears this time sequence in mind (Sitnov et al. 2009). In the data the jet front is identified as a sharp increase in the magnetic vertical component and is called the dipolarization front. Runov et al. (2009), using data from an interval when four THEMIS spacecraft were aligned along the tail-axis, reported observations of a depolarization front detected in the central plasma sheet sequentially at $X=-20.1 \mathrm{R}_{\mathrm{e}},-16.7 \mathrm{R}_{\mathrm{e}}$ and at $-11.0 \mathrm{R}_{\mathrm{e}}$. The similarity of the observed magnetic profile suggested that the jet-front remained coherent as it traveled at a velocity of $300 \mathrm{~km} / \mathrm{s}$ over a distance more than $10 \mathrm{R}_{\mathrm{e}}$ (Fig. 5). The study provided the first unambiguous evidence of the earthward propagation of a dipolarization front. The front separated different plasma populations and its thickness was found to be as small as the ion inertial length. THEMIS spacecraft observations of a dipolarization front at $X=-9--11 \mathrm{R}_{\mathrm{e}}$ near midnight and close to the neutral sheet showed that the front was a narrow interface between plasmas of different densities and temperatures and contained a very thin current layer (Sergeev et al. 2009). A short-lived depression of the total magnetic field devoid of wave activity was also detected ahead of the front. The sub-ion-gyroradius thickness of the front made finite gyroradius effects show up clearly. Furthermore strong electric field activity in the lower-hybrid range was detected. These observations indicate that rich kinetic effects are induced at the interface where a reconnection jet interacts with the plasma at rest ahead of it.

The dynamic behavior of the jet front was predicted by hybrid simulations of Nakamura et al. (2002). Around the interface between the reconnection jet and the pre-existing plasma sheet ahead of it, the curved reconnected field lines are piled up on the jet side. On the other side of the interface, the pre-existing plasma density is enhanced by compression. Then the interface becomes unstable to the interchange instability. The wavy deformation of the interface grows non-linearly into magnetic bubbles into which the reconnected field lines converge (Fig. 6). The bubbles, which move faster than the other parts, can sustain a localized flow channel with a width of a few Earth radii, in which plasma and magnetic field transport are achieved. TanDokoro and Fujimoto (2005) showed that the jet front was unstable in the MHD system as well. Recent observations showed that the jet front appeared recurrently (Zhou et al. 2009a, 2009b). Possible explanations are that these recurrent appearances reflect multiple jet ejection or that they are due to the spacecraft's traversal of a highly structured front. Guzdar et al. (2010) showed that the depolarization front could deform itself into a mushroom shape and proposed that the multiple sharp magnetic jumps seen in the time-series data were reproduced in a virtual observation along a cut through the mushroom-shaped front. 
Fig. 5 Magnetic field data from four THEMIS spacecraft aligned along the tail-axis observing a depolarization front sequentially at $X=-20.1 \mathrm{R}_{\mathrm{e}},-16.7 \mathrm{R}_{\mathrm{e}}$ and at $-11.0 \mathrm{R}_{\mathrm{e}}$. These provide the first unambiguous evidence of the earthward propagation of a dipolarization front. From Runov et al. (2009)

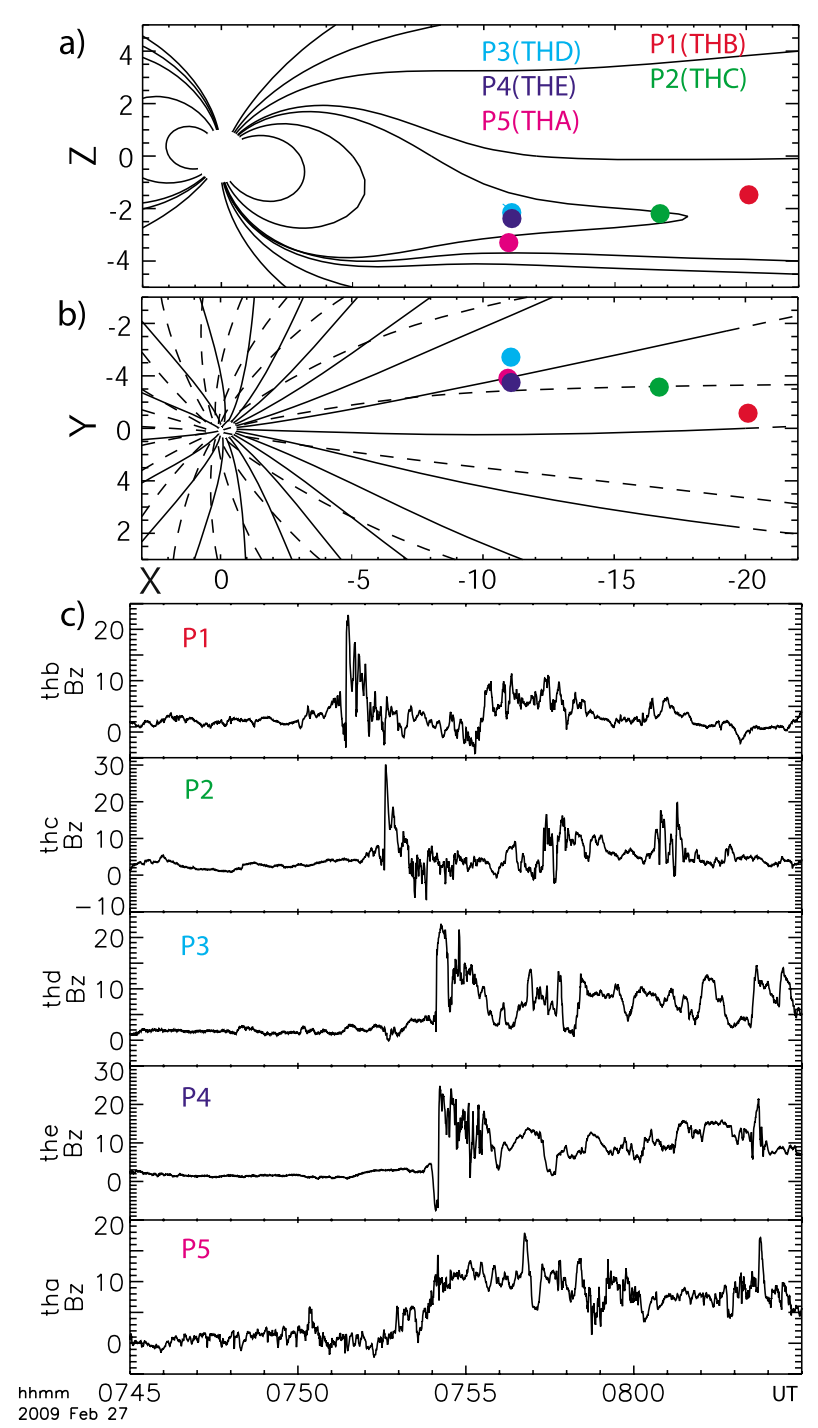

When there are multiple $\mathrm{X}$-lines, an X-line interacts with neighboring X-lines. The immediate product of the interaction is magnetic islands. Then the islands are subject to merging process. While this is well known from simulations using periodic boundary conditions, it is a good challenge for an observer to show smoking gun evidence of the coalescence process. The meso-scale magnetic island described in Retino et al. (2008) could be the one. In the middle of a magnetic island, whose lateral scale was $\sim 10$ times the ion inertial length, a dip in density and a peak in the guide field component were observed to be co-located. Comparison with two-dimensional full particle simulation results showed that simultaneous detection of the two signatures was possible at the center of a merged island shortly after the completion of merging, and that, if the two smaller islands subject to the merging had been well matured to have their outermost part to be coated with lobe field lines. The same island event has been further studied with emphasis on plasma wave features (Khotyaintsev et al. 


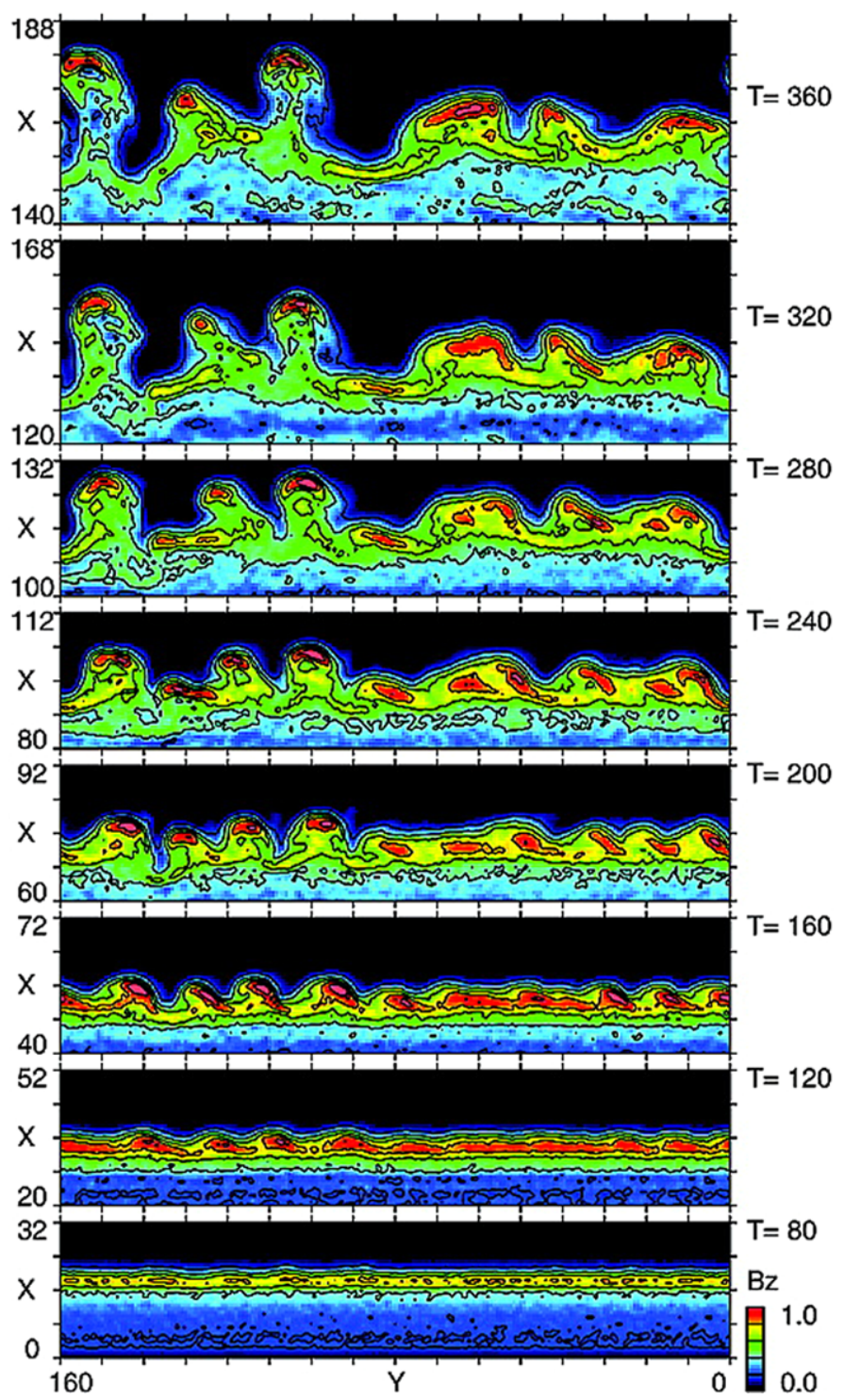

Fig. 6 The jet-front interface is unstable to the interchange instability, which grows non-linearly to form magnetic bubbles. From Nakamura et al. (2002)

2010). Two different types of waves, both indicative of dynamic behavior of electrons inside the meso-scale magnetic island, were observed. One type was the slow ESWs, which were likely to be produced by Buneman instability. The slow ESWs were observed in the center of the magnetic island (Fig. 7). While the X-line facilitating the merging was not directly encountered, the slow ESWs were detected shortly after the X-line had disappeared. In the lack of any better idea, it was proposed that the slow ESWs were excited by Buneman instability at the merging X-line and somehow survived until the detection by the Cluster-II spacecraft. 
Fig. 7 The wave form of slow ESWs, which are likely to be produced by Buneman instability, observed at the center of a meso-scale magnetic island shortly after the merging process that produced the island itself. From Khotyaintsev et al. (2010)
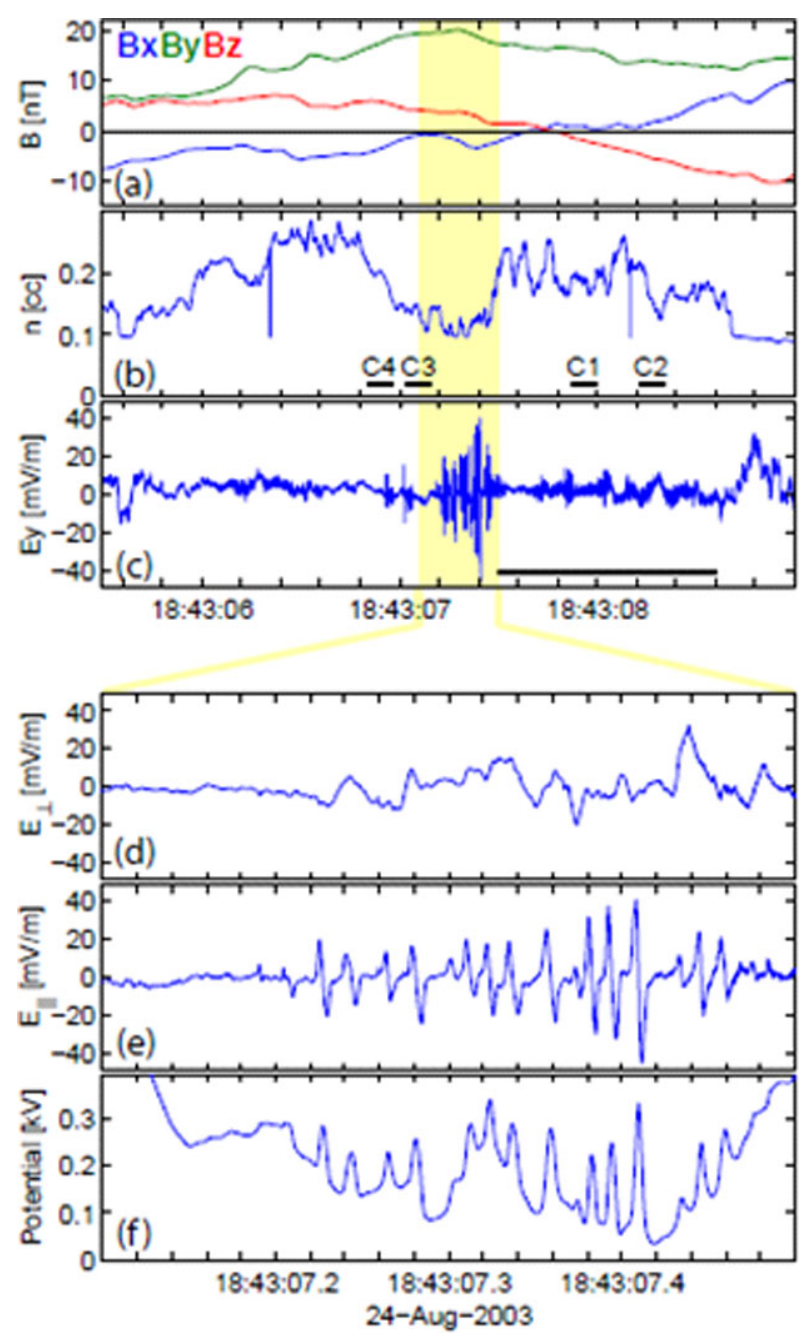

This implies that the merging X-line involved a current density which was intense enough to satisfy the instability condition.

Recent full-particle simulation studies have shown that magnetic island coalescence is an efficient way of producing energetic electrons (Oka et al. 2010; Tanaka et al. 2010). Simulated in these studies was the production of multiple magnetic islands and their merging in an extended current sheet. It was shown that a merging at later phases forming a large island produced the most energetic electron population in the system. The strong electron acceleration was because the reverse-reconnection facilitating the merging was strongly driven. The associated electric field turned out be an order of magnitude larger than those available upon normal reconnection. Drake et al. (2006) proposed another way by which magnetic islands can contribute to particle acceleration. In their simulation, particles were accelerated when an island reduced its size. Such a process is expected to occur in a sequence of magnetic island coalescence. These results suggest a non-negligible role played by magnetic islands in particle acceleration. Significant difference in the degree of particle acceleration between a 
single X-line case (which is more or less laminar) and a multiple $\mathrm{X}$-line case (which is more or less turbulent) would be expected. Chen et al. (2008) reported a magnetotail reconnection event that would indicate a possible link between energetic electrons and magnetic islands.

\section{Discussion}

Wave data are very eloquent in revealing the micro-scale dynamics that is not resolved by particle data or that too localized for a spacecraft to have reasonable chance to make a direct encounter with it. A good example is the recent identification of slow ESWs excited by Buneman instability. The instability does require a very thin current sheet. While formation flying observations available so far have not identified such a thin current layer from multipoint observations of magnetic field, the detection of the Buneman ESW does prove that such an extremely intense current density is indeed generated. In view of the highly transient nature of slow ESWs seen in the initial value problem (a particle simulation starting from an unstable current sheet isolated from its environment), a real case that lets ESWs to appear continuously and to be detected by a spacecraft should be in a driven situation where the current sheet is pinched severely by the surrounding large scale dynamics for a certain time duration.

More detailed particle and wave data will be available from future missions. We, however, do not yet have a framework to fully appreciate the information to be obtained from these spacecraft observations. In evaluating feedback effects of waves to a parent larger scale process, what is widely done today is to estimate the transport coefficients using a quasi-linear theory. Meanwhile, non-linear effects are at least visible in the waveform data. In quantifying non-linear effects it would be required to refer to numerical simulation results. On the other hand, to perform a full-particle simulation with all the parameters being equal to a real situation (the mass ratio, the ratio between the electron plasma frequency and the electron cyclotron frequency, and so on) is very difficult and will remain so for a multi-dimensional case in the foreseeable future. Here we face the need to develop a new scheme of data-simulation synergistic studies.

What we have learned in the QMRT case described in a previous section is that wave effects are not only non-linear but can be non-local at the same time. In QMRT, non-linear wave effects at the edges of a current sheet induced current density enhancement and/or electron temperature anisotropization at the center of the current sheet remote from where the waves were excited. Then the new current sheet structure was vulnerable to the quickly growing tearing mode instability. Note that wave activity was absent and "anomalous resistivity" was almost zero at the neutral sheet. Simultaneous multi-scale observations by a well coordinated spacecraft fleet are necessary to evaluate such a non-local effect in the real space.

The majority of full particle simulation studies of reconnection to date isolate the X-line and try to elucidate its physics. On the other hand, we have seen that magnetic island coalescence plays a substantial role in electron acceleration. Data in the magnetotail indicate that a large fraction of the X-lines are moving tailward, which should not be the case if an $\mathrm{X}$-line is isolated. We have also seen that a jet emanated from an X-line does not propagate freely towards "an open boundary," but its front interact, with the plasma at rest ahead of it, with the interaction process possessing rich physics. One may generalize this list of concrete issues to state that more attention should be paid to studying how an X-line interacts with those surrounding it. 
Almost all the studies on waves in reconnection assume two-dimensional reconnection geometry and then add a small degree of freedom in the third dimension to allow a wave activity. In this regard, little is known about full three-dimensional effects in the full presence of kinetic effects. Again, since it will remain very difficult to perform a full-particle simulation with real parameters in the foreseeable future, we face the need to develop a scheme for re-scaling the simulation results. This is a critical point for future studies because when the steady, laminar, two-dimensional picture of magnetic reconnection is about to stop being the standard picture, the importance of simulation studies will be enhanced substantially.

Acknowledgements The authors would like to acknowledge the discussion at the Yosemite meeting, with M. Oka, and with the team members of the ISSI working group (Lead: Y. Khotyaintsev).

Open Access This article is distributed under the terms of the Creative Commons Attribution Noncommercial License which permits any noncommercial use, distribution, and reproduction in any medium, provided the original author(s) and source are credited.

\section{References}

M. André, A. Vaivads, S.C. Buchert, A.N. Fazakerley, A. Lahiff, Thin electron-scale layers at the magnetopause. Geophys. Res. Lett. 31, L03803 (2004). doi:10.1029/2003GL018137

Y. Asano, T. Mukai, M. Hoshino, Y. Saito, H. Hayakawa, T. Nagai, Statistical study of thin current sheet evolution around substorm onset. J. Geophys. Res. 109, A05213 (2004). doi:10.1029/2004JA010413

Y. Asano, R. Nakamura, W. Baumjohann, A. Runov, Z. Voros, M. Volwerk, T.L. Zhang, A. Balogh, B. Klecker, H. Reme, How typical are atypical current sheets? Geophys. Res. Lett. 32, L03108 (2005). doi:10.1029/2004GL021834

S.D. Bale, F.S. Mozer, T. Phan, Observation of lower hybrid drift instability in the diffusion region at a reconnecting magnetopause. Geophys. Res. Lett. 29(24), 2180 (2002). doi:10.1029/2002GL016113

J.U. Brackbill, D.W. Forslund, K.B. Quest, D. Winske, Nonlinear evolution of the lower-hybrid drift instability. Phys. Fluids 27, 2682-2693 (1984)

T.A. Carter, H. Ji, F. Trintchouk, M. Yamada, R.M. Kulsrud, Measurement of lower-hybrid drift turbulence in a reconnecting current sheet. Phys. Rev. Lett. 88, 015001-1 (2002)

C.A. Cattell, F.S. Mozer, Experimental determination of the dominant wave mode in the active near-earth magnetotail. Geophys. Res. Lett. 13, 221-224 (1986)

C.A. Cattell, F.S. Mozer, Substorm-associated lower-hybrid waves in the plasma sheet observed by ISEE-1, in Magnetotail Physics, ed. by A.T.Y. Lui (John Hopkins University Press, Baltimore, 1987), p. 119

C.A. Cattell, M. Kim, R.P. Lin, F.S. Mozer, Observations of large electric fields near the plasma sheet boundary by ISEE-1. Geophys. Res. Lett. 9, 539-542 (1982)

C.A. Cattell, et al. Cluster observations of electron holes in association with magnetotail reconnection and comparison to simulations. J. Geophys. Res. 110, A01211 (2005). doi:10.1029/2004JA010519

C.C. Chaston, T.D. Phan, J.W. Bonnell, F.S. Mozer, M. Acuńa, M.L. Goldstein, A. Balogh, M. Andre, H. Reme, A. Fazakerley, Drift-kinetic Alfvén waves observed near a reconnection X line in the Earth's magnetopause. Phys. Rev. Lett. 95, 065002 (2005)

C.C. Chaston, J.R. Johnson, M. Wilber, M. Acuna, M.L. Goldstein, H. Reme, Kinetic Alfven wave turbulence and transport through a reconnection diffusion region. Phys. Rev. Lett. 102, 015001 (2009)

H. Che, J.F. Drake, M. Swisdak, P.H. Yoon, Electron holes and heating in the reconnection dissipation region. Geophys. Res. Lett. 37, L11105 (2010). doi:10.1029/2010GL043608

L.-J. Chen et al., Evidence of an extended electron current sheet and its neighboring magnetic island during magnetotail reconnection. J. Geophys. Res. 113, A12213 (2008a). doi:10.1029/2008JA013385

L.-J. Chen et al., Observation of energetic electrons within magnetic islands. Nat. Phys. 4, 19-23 (2008b)

F.V. Coroniti, A. Eviatar, Magnetic field reconnection in a collisionless plasma. Astrophys. J. Suppl. Ser. 33, 189-210 (1977)

W. Daughton, Two-fluid theory of the drift kink instability. J. Geophys. Res. 104, 28701 (1999)

W. Daughton, Electromagnetic properties of the lower-hybrid drift instability in a thin current sheet. Phys. Plasmas 10, 3103 (2003)

W. Daughton, G. Lapenta, P. Ricci, Nonlinear evolution of the lower-hybrid drift instability in a current sheet. Phys. Rev. Lett. 93 (2004). doi:10.1103/93.105004 
W. Daughton, V. Roytershteyn, H. Karimabadi, L. Yin, B.J. Albright, B. Bergen, K.J. Bowers, Role of electron physics in the development of turbulent magnetic reconnection in collisionless plasmas. Nat. Phys. (2011). doi:10.1038/NPHYS1965

R.C. Davidson, N.T. Gladd, C.S. Wu, J.D. Huba, Effects of finite plasma beta on the lower-hybrid-drift instability. Phys. Fluids 20, 301-310 (1977)

X.H. Deng, H. Matsumoto, Rapid magnetic reconnection in the Earth's magnetosphere mediated by whistler waves. Nature 410, 557-560 (2001)

X.H. Deng, H. Matsumoto, H. Kojima, T. Mukai, R.R. Anderson, W. Baumjohann, R. Nakamura, Geotail encounter with reconnection diffusion region in the Earth's magnetotail: evidence of multiple X lines collisionless reconnection? J. Geophys. Res. 109 (2004). doi:10.1029/2003JA010031

J.F. Drake, M. Swisdak, C. Cattell, M.A. Shay, B.N. Rogers, A. Zeiler, Formation of electron holes and particle energization during magnetic reconnection. Science 299, 873 (2003)

J.F. Drake, M. Swisdak, H. Che, M.A. Shay, Electron acceleration from contracting magnetic islands during reconnection. Nature 443, 553 (2006)

J.P. Eastwood, T.D. Phan, S.D. Bale, A. Tjulin, Observations of turbulence generated by magnetic reconnection. Phys. Rev. Lett. 102, 035001 (2009)

R.E. Ergun, L. Andersson, J. Tao, V. Angelopoulos, J. Bonnell, J.P. McFadden, D.E. Larson, S. Eriksson, T. Johansson, C.M. Cully, D.N. Newman, M.V. Goldman, A. Roux, O. LeContel, K.-H. Glassmeier, W. Baumjohann, Observations of double layers in Earth's plasma sheet. Phys. Rev. Lett. 102, 155002 (2009)

W.M. Farrell, M.D. Desch, M.L. Kaiser, K. Goetz, The dominance of electron plasma waves near a reconnection X-line region. Geophys. Res. Lett. 29 (2002). doi:10.1029/2002GL014662

K. Fujimoto, S. Machida, A generation mechanism of electrostatic waves and subsequent electron heating in the plasma sheet-lobe boundary region during magnetic reconnection. J. Geophys. Res. 111, A09216 (2006). doi:10.1029/2005JA011542

D.A. Gurnett, L.A. Frank, R.P. Lepping, Plasma waves in the distant magnetotail. J. Geophys. Res. 81, 60596071 (1976)

P.N. Guzdar, A.B. Hassam, M. Swisdak, M.I. Sitnov, A simple MHD model for the formation of multiple dipolarization fronts. Geophys. Res. Lett. 37, L20102 (2010). doi:10.1029/2010GL045017

G. Haerendel, Microscopic plasma processes related to reconnection. J. Atmos. Sol.-Terr. Phys. 40, 343-353 (1978)

M. Hesse, M. Kuznetsova, M. Hoshino, The structure of the dissipation region for component reconnection: particle simulations. Geophys. Res. Lett. 29(12), 1563 (2002). doi:10.1029/2001GL014714

J.D. Huba, N.T. Gladd, K. Papadopoulos, The lower-hybrid-drift instability as a source of anomalous resistivity for magnetic field line reconnection. Geophys. Res. Lett. 4, 125-128 (1977)

J.D. Huba, N.T. Gladd, K. Papadopoulos, Lower-hybrid-drift wave turbulence in the distant magnetotail. J. Geophys. Res. 81, 5217-5226 (1978)

J.D. Huba, J.F. Drake, N.T. Gladd, Lower-hybrid-drift instability in field reversed plasmas. Phys. Fluids 23, 552-561 (1980)

H. Ji, S. Terry, M. Yamada, R. Kulsrud, A. Kuritsyn, Y. Ren, Electromagnetic fluctuations during fast reconnection in a laboratory plasma. Phys. Rev. Lett. 92, 115001-1 (2004)

J.R. Johnson, C.Z. Cheng, Kinetic Alfvén waves and plasma transport at the magnetopause. Geophys. Res. Lett. 24, 1423 (1997)

H. Karimabadi, W. Daughton, K.B. Quest, Role of electron temperature anisotropy in the onset of magnetic reconnection. Geophys. Res. Lett. 31 (2004). doi:10.1029/2004GL020791

Y.V. Khotyaintsev, A. Vaivads, M. Andre, M. Fujimoto, A. Retino, C.J. Owen, Observations of slow electron holes at a magnetic reconnection site. Phys. Rev. Lett. 105, 165002 (2010)

H. Kojima, H. Matsumoto, S. Chikuba, S. Horiyama, M. Ashour-Abdalla, R.R. Anderson, GEOTAIL waveform observations of broadband/narrowband electrostatic noise in the distant tail. J. Geophys. Res. 102, $14439-14455$ (1997a)

H. Kojima, H. Furuya, H. Usui, H. Matsumoto, Modulated electron plasma waves observed in the tail lobe: GEOTAIL waveform observations. Geophys. Res. Lett. 24, 3049-3052 (1997b)

H. Kojima, Y. Omura, H. Matsumoto, K. Miyaguti, T. Mukai, Characteristics of electrostatic solitary waves observed in the plasma sheet boundary: statistical analyses. Nonlinear Process. Geophys. 6, 179-186 (1999)

N.A. Krall, P.C. Liewer, Low frequency instabilities in magnetic pulses. Phys. Rev. A 4, 2094-2103 (1971)

M.M. Kuznetsova, M. Hesse, L. Rastätter, A. Taktakishvili, G. Toth, D.L. De Zeeuw, A. Ridley, T.I. Gombosi, Multiscale modeling of magnetospheric reconnection. J. Geophys. Res. 112, A10210 (2007). doi:10.1029/2007JA012316

A.T.Y. Lui, A. Mankofsky, C.-L. Chang, K. Papadopoulos, C.S. Wu, A current disruption mechanism in the neutral sheet: a possible trigger for substorm expansions. Geophys. Res. Lett. 17, 745-748 (1990) 
H. Matsumoto, I. Nagano, R.R. Anderson, H. Kojima, K. Hashimoto, M. Tsutsui, T. Okada, I. Kimura, Y. Omura, M. Okada, Plasma wave observations with GEOTAIL spacecraft. J. Geomagn. Geoelectr. 46, 59-95 (1994a)

H. Matsumoto, H. Kojima, T. Miyatake, Y. Omura, M. Okada, I. Nagano, M. Tsutsui, Electrostatic Solitary Waves (ESW) in the Magnetotail: BEN wave forms observed by GEOTAIL. Geophys. Res. Lett. 21, 2915-2918 (1994b)

H. Matsumoto, X.H. Deng, H. Kojima, R.R. Anderson, Observation of electrostatic solitary waves associated with reconnection on the dayside magnetopause boundary. Geophys. Res. Lett. 30 (2003). doi:10.1029/2002GL016319

F.S. Mozer, S.D. Bale, T.D. Phan, Phys. Rev. Lett. 89, 015002 (2002)

T. Nagai, M. Fujimoto, R. Nakamura, Y. Saito, T. Mukai, T. Yamamoto, A. Nishida, S. Kokubun, G.D. Reeves, R.P. Lepping, Geotail observations of a fast tailward flow at XGSM $=-15$ Re. J. Geophys. Res 103, 23543-23550 (1998)

T. Nagai, I. Shinohara, M. Fujimoto, M. Hoshino, Y. Saito, S. Machida, T. Mukai, Geotail observations of the Hall current system: evidence of magnetic reconnection in the magnetotail. J. Geophys. Res. 106(A11), 25,929-25,949 (2001)

T. Nagai, I. Shinohara, M. Fujimoto, A. Matsuoka, Y. Saito, T. Mukai, Construction of magnetic reconnection in the near-Earth magnetotail with Geotail. J. Geophys. Res. 116, A04222 (2011). doi:10.1029/2010JA016283

M.S. Nakamura, H. Matsumoto, M. Fujimoto, Interchange instability at the leading part of reconnection jets. Geophys. Res. Lett. 29(8), 1247 (2002). doi:10.1029/2001GL013780

R. Nakamura, W. Baumjohann, Y. Asano, A. Runov, A. Balogh, C.J. Owen, A.N. Fazakerley, M. Fujimoto, B. Klecker, H. Rème, Dynamics of thin current sheets associated with magnetotail reconnection. J. Geophys. Res. 111, A11206 (2006). doi:10.1029/2006JA011706

M. Oka et al., Astrophys. J. 714, 915 (2010)

T. Okada, K. Tsuruda, H. Hayakawa, M. Nakamura, T. Mukai, T. Yamamoto, A. Matsuoka, T. Terasawa, F.S. Mozer, S. Kokubun, H. Matsumoto, GEOTAIL observations of electrostatic waves in the lower hybrid frequency range in the plasma sheet boundary layer. Geophys. Res. Lett. 21, 2931-2934 (1994)

Y. Omura, H. Kojima, H. Matsumoto, Computer simulation of electrostatic solitary waves: a nonlinear model of broadband electrostatic noise. Geophys. Res. Lett. 21, 2923-2926 (1994)

Y. Omura, H. Matsumoto, T. Miyake, H. Kojima, Electron beam instabilities as generation mechanism of electrostatic solitary waves in the magnetotail. J. Geophys. Res. 101, 2685-2697 (1996)

T.G. Onsager, M.F. Thomsen, R.C. Elphic, J.T. Gosling, R.R. Anderson, G. Kettmann, Electron generation of electrostatic waves in the plasma sheet boundary layer. J. Geophys. Res. 15, 15509-15519 (1993)

G.K. Parks, M. McCarthy, R.J. Fitzenreiter, J. Etcheto, K.A. Anderson, R.R. Anderson, T.E. Eastman, L.A. Frank, D.A. Gurnett, C. Huang, R.P. Lin, A.T.Y. Lui, K.W. Oglivie, A. Pedersen, H. Reme, D.J. Williams, Particle and field characteristics of the high-latitude plasma sheet boundary layer. J. Geophys. Res. 89, 8885-8906 (1984)

P. Petkaki, M.P. Freeman, A.P. Walsh, Cluster observations of broadband electromagnetic waves in and around a reconnection region in the Earth's magnetotail current sheet. Geophys. Res. Lett. 33 (2006). doi:10.1029/2006GL2006GL027066

A. Retino et al., Cluster observations of energetic electrons and electromagnetic fields within a reconnecting thin current sheet in the Earth's magnetotail. J. Geophys. Res. 113, A12215 (2008). doi:10.1029/2008JA013511

P. Ricci, U. Brackbill, W. Daughton, G. Lapenta, Influence of the lower hybrid drift instability on the onset of magnetic reconnection. Phys. Plasmas 11, 4489 (2004)

A. Runov, V. Angelopoulos, M.I. Sitnov, V.A. Sergeev, J. Bonnell, J.P. McFadden, D. Larson, K.-H. Glassmeier, U. Auster, THEMIS observations of an earthwardpropagating dipolarization front. Geophys. Res. Lett. 36, L14106 (2009). doi:10.1029/2009GL038980

F.L. Scarf, L.A. Frank, K.L. Ackerson, R.P. Lepping, Plasma wave turbulence at distant crossings of the plasma sheet boundaries and the neutral sheet. Geophys. Res. Lett. 1, 189-192 (1974)

M. Scholer, I. Sidorenko, C.H. Jaroschek, R.A. Treumann, A. Zeiler, Onset of collisionless magnetic reconnection in thin current sheet. Phys. Plasmas 10(9), 3521-3527 (2003)

V. Sergeev, V. Angelopoulos, S. Apatenkov, J. Bonnell, R. Ergun, R. Nakamura, J. McFadden, D. Larson, A. Runov, Kinetic structure of the sharp injection/dipolarization front in the flow-braking region. Geophys. Res. Lett. 36, L21105 (2009). doi:10.1029/2009GL040658

I. Shinohara, T. Nagai, M. Fujimoto, T. Terasawa, T. Mukai, K. Tsuruda, T. Yamamoto, Low-frequency electromagnetic turbulence observed near the substorm onset site. J. Geophys. Res. 103(A9), 2036520388 (1998)

I. Shinohara, M. Fujimoto, in Frontiers of Magnetospheric Physics. COSPAR Colloquium Ser. vol. 16, ed. by M. Hoshino, Y. Omura, L.J. Lanzerotti (Elsevier, Amsterdam, 2005), p. 123 
M.I. Sitnov, P.N. Guzdar, M. Swisdak, A model of the bifurcated current sheet. Geophys. Res. Lett. 30(13), 1712 (2003). doi:10.1029/2003GL017218

M.I. Sitnov, M. Swisdak, A.V. Divin, Dipolarization fronts as a signature of transient reconnection in the magnetotail. J. Geophys. Res. 114, A04202 (2009). doi:10.1029/2008JA013980

K.G. Tanaka, I. Shinohara, M. Fujimoto, Quick magnetic reconnection triggering in an ion-scale current sheet: transition from Type-I to Type-II. Geophys. Res. Lett. 32 (2005). doi:10.1029/2005GL023718

K.G. Tanaka, T. Yumura, M. Fujimoto, I. Shinohara, S.V. Badman, A. Grocott, Merging of magnetic islands as an efficient accelerator of electrons. Phys. Plasmas 17, 102902 (2010). doi:10.1063/1.3491123

M. Tanaka, T. Sato, Simulations on lower hybrid drift instability and anomalous resistivity in the magnetic neutral sheet. J. Geophys. Res. 86, 5541-5552 (1981)

R. TanDokoro, M. Fujimoto, Three-dimensional MHD simulation study of the structure at the leading part of a reconnection jet. Geophys. Res. Lett. 32, L23102 (2005). doi:10.1029/2005GL024467

T. Umeda, Y. Omura, H. Matsumoto, H. Usui, Formation of electrostatic solitary waves in space plasmas: particle simulations with open boundary conditions. J. Geophys. Res. 107(A12), 1449 (2002). doi:10.1029/2001JA000286

X.H. Wei, J.B. Cao, G.C. Zhou, O. Santlick, H. Reme, I. Dandouras, N. Cornilleau-Wehrlin, E. Lucek, C.M. Carr, A. Fazakerley, J. Geophys. Res. 112 (2007). doi:10.1029/2006JA011771

D. Winske, P.C. Liewer, Particle simulation studies of the lower hybrid drift instability. Phys. Fluids 21, 1017-1025 (1978)

J.R. Wygant et al., Cluster observations of an intense normal component of the electric field at a thin reconnecting current sheet in the tail and its role in the shock-like acceleration of the ion fluid into the separatrix region. J. Geophys. Res. 110, A09206 (2005). doi:10.1029/2004JA010708

P. Yoon, A. Lui, M. Sitnov, Phys. Plasmas 9, 1526 (2002)

M. Zhou, X.H. Deng, S.Y. Li, Y. Pang, A. Vaivads, H. Rème, E. Lucek, S. Fu, X. Lin, Z.G. Yuan, J.F. Wang, Observation of waves near lower hybrid frequency in the reconnection region with thin current sheet. J. Geophys. Res. 114 (2009a). doi:10.1029/2008JA013427

X.-Z. Zhou et al., Thin current sheet in the substorm late growth phase: modeling of THEMIS observations. J. Geophys. Res. 114, A03223 (2009b). doi:10.1029/2008JA013777 